\title{
Estimation of Interference-Free Transmit Power for Opportunistic Spectrum Access
}

\author{
Brian L. Mark and Ahmed O. Nasif \\ Dept. of Electrical and Computer Engineering \\ George Mason University, Fairfax, VA
}

\begin{abstract}
We consider a scenario in which frequency agile radios opportunistically share a fixed spectrum resource with a set of primary nodes. We develop a collaborative scheme for frequency agile radios to estimate the maximum power at which they can transmit, without causing harmful interference to the primary receivers. The proposed scheme relies on signal strength measurements, which are used to localize primary transmitters. An approximation to the maximum interference-free transmit power is derived using the Cramér-Rao lower bound on localization accuracy. We present numerical results to demonstrate the effectiveness of the proposed scheme under a variety of scenarios.
\end{abstract}

\section{INTRODUCTION}

In conventional wireless systems, the spectrum is allocated statically among a set of transmitters over a geographic coverage area. Recent studies have shown that significant portions of the wireless spectrum are highly underutilized over certain geographic coverage areas [1]. In principle, such "spectrum holes" could be exploited by frequency-agile radios (FARs), which are capable of dynamically tuning to different frequency ranges. Frequency agility and high receiver sensitivity are key features of emerging cognitive radios [2]. An open research question is whether effective opportunistic spectrum sharing can be realized efficiently and practically.

An essential first step in opportunistic spectrum access for a FAR node is to determine the maximum power with which it can transmit on a given frequency channel without causing harmful interference to primary users. In this paper ${ }^{1}$, we develop a method to estimate the maximum interference-free transmit power (MIFTP) for a FAR node on a given radio channel, based on signal strength measurements. The signal strength measurements may be obtained by a single FAR node at different locations at different points of time, or by collaborative sharing of measurement information among spatially separated FAR nodes. We develop an estimator for the location of the primary transmitter and an associated Cramér-Rao bound (CRB) on the error of the estimator. An estimate for the MIFTP is then obtained using the $\mathrm{CRB}$ for the location estimator. We assume that the primary node transmits at constant power during an observation period; we do not address the separate issue of opportunistic spectrum access in the time-domain, i.e.,

\footnotetext{
${ }^{1}$ This work was supported in part by the National Science Foundation under Grants CNS-0520151 and ECS-0246925.
}

exploiting periods for which the primary transmitter may be idle [3], [4].

In [5], the impact of secondary transmissions on a primary receiver is studied in terms of interference probability. Because of the integral forms involved it is difficult to use the given probability expressions to solve for (even homogeneous) allowable secondary transmit power. In [6], an additional no-talk radius is defined within which the secondary users must be quiet to guarantee service to primary users within some a protected radius. Once these distances are specified (in terms of SNR margins), the aggregate interference at the edge of the protected region is computed, which can then be used to obtain the total permissible secondary transmit power. However, this approach assumes that the primary transmit power and the local SNR at the secondary receivers are already known, so that SNR can be used as a proxy for distance. To avoid these limitations, our approach exploits collaboration among secondary nodes for explicit sensing of the primary transmitter's power and location.

The remainder of the paper is organized as follows. Section II describes our assumed model for opportunistic spectrum sharing. Section III discusses signal strengthbased localization and the associated CRB. Section IV derives an approximation for the MIFTP. Section V presents numerical results, which demonstrate the accuracy of the approximation. Finally, the paper is concluded in Section VI.

\section{Model for Opportunistic Spectrum Access}

\section{A. Signal strength model}

We assume that all transmissions are omnidirectional and the signal propagation is governed by a lognormal shadowing model (cf. [7]) such that the propagation loss between two nodes $i$ and $j$ is given by

$$
L_{i, j}=g\left(d_{i, j}, \epsilon\right)+W
$$

where the function $g(d, \epsilon)=10 \epsilon \log _{10} d$ represents the generic log-distance path loss component, with $\epsilon$ denoting the path loss factor. For simplicity, we denote $g(d, \epsilon)$ by $g(d)$. We assume that the shadowing noise $W \sim$ $\mathcal{N}\left(0, \sigma_{W}^{2}\right)$. If the receiver noise power, antenna gains, etc., are known and the fast-fading effects are sufficiently averaged out, then the received signal strength (SS) at node $i$ due to node $j$ is given by

$$
S_{i}=s_{j}-L_{i, j}=s_{j}-g\left(d_{i, j}\right)+W,
$$


where $s_{j}$ is the transmit power of node $j$.

\section{B. Maximum interference-free transmit power}

Consider a FAR node $a$ and a primary transmitter $p$, which transmits on a given frequency channel $\gamma$. For the FAR node $a$ to use the channel $\gamma$ opportunistically, it must determine the maximum power at which it can transmit on channel $\gamma$, without causing harmful interference to any potential receiver or victim node $v$ within the range of the primary transmitter $p$. More formally, the maximum interference-free transmit power (MIFTP) on a frequency channel $\gamma$ is defined as the maximum transmit power of node $a$ on channel $\gamma$, such that the probability of interference to any victim node $v$ is less than a prescribed threshold (cf. [8]).

Thus, the MIFTP determines the radius of a spectrum hole considered in the spatial domain, centered at the FAR node $a$, with respect to a given frequency channel $\gamma$. For the channel $\gamma$ to be usable for FAR node $a$, its associated MIFTP must be sufficiently large to allow communications with at least one other FAR node. The issue of how FAR nodes opportunistically select frequency channels to form a wireless network of FAR nodes is an important topic that is beyond the scope of the present work. In this paper, we focus on the problem of accurately estimating the MIFTP for a given FAR node, which is a critical enabler of opportunistic spectrum sharing.

The received power at node $v$ due to node $p$ is given by

$$
R_{v}=s_{p}-L_{p, v}=s_{p}-g\left(d_{p, v}\right)+W,
$$

where $s_{p}$ is the transmit power of node $p$. The received power at node $v$ from node $a$ is given by

$$
I_{v}=s_{a}-L_{a, v}=s_{a}-g\left(d_{a, v}\right)+W,
$$

where $s_{a}$ is the transmit power of $a$.

We define the outage probability of a victim node $v$ with respect to the transmitter $p$, as the probability that the received power $R_{v}$ from node $p$ is below a predefined detection threshold $r_{\min }$ :

$$
P_{\text {out }}(p, v) \triangleq P\left\{R_{v}<r_{\min }\right\},
$$

when $p$ is transmitting. The coverage distance is defined as the maximum distance between the node $p$ and any potential victim node $v$ such that the outage probability does not exceed a predefined threshold $\varepsilon_{\mathrm{cov}}>0$ :

$$
\begin{aligned}
d_{\mathrm{cov}}(p) & \triangleq \max \left\{d_{p, v}: P_{\mathrm{out}}(p, v) \leq \varepsilon_{\mathrm{cov}}\right\} \\
& =g^{-1}\left(s_{p}-r_{\mathrm{min}}+\sigma_{W} Q^{-1}\left(1-\varepsilon_{\mathrm{cov}}\right)\right),
\end{aligned}
$$

where $g^{-1}(\cdot)$ denotes the inverse of $g(\cdot)$ and $Q(x) \triangleq$ $\frac{1}{\sqrt{2 \pi}} \int_{x}^{\infty} e^{-\frac{t^{2}}{2}} d t$ denotes the standard $Q$-function. Note that $d_{\mathrm{cov}}(p)$ depends on $s_{p}, r_{\min }, \varepsilon_{\mathrm{cov}}, \sigma_{W}^{2}$, and the path loss function $g(\cdot)$. We assume that the FAR node knows or can estimate $s_{p}$ and therefore can evaluate $d_{\text {cov }}(p)$. The circle centered at node $p$ with radius $d_{\text {cov }}(p)$ is called the coverage area of the transmitter $p$. Any potential victim node $v$, which lies outside of coverage area of node $p$ would be oblivious to the interference caused by the FAR node $a$.

We define the interference probability with respect to a given victim node $v$ as the probability that $I_{v}$ exceeds a predefined interference tolerance threshold $i_{\max }$ :

$$
P_{\text {int }}(a, v) \triangleq \operatorname{Pr}\left\{I_{v} \geq i_{\max }\right\}
$$

when node $a$ is transmitting. For a fixed primary transmitter $p$ and FAR node $a$, the MIFTP is defined as the maximum transmit power of the FAR node such that the interference probability with respect to any potential victim node within the coverage distance from node $p$ does not exceed a threshold $\varepsilon_{\text {int }}>0$ :

$$
s_{a}^{*} \triangleq \max \left\{s_{a}: P_{\text {int }}(a, v) \leq \varepsilon_{\text {int }}, \forall v: d_{p, v} \leq d_{\text {cov }}(p)\right\} .
$$

Proposition 1: If $d_{p, a}>d_{\mathrm{cov}}(p)$, the MIFTP is given by

$$
s_{a}^{*}=i_{\max }+g\left(d_{a}^{*}\right)-\sigma_{W} Q^{-1}\left(\varepsilon_{\mathrm{int}}\right),
$$

where $d_{a}^{*} \triangleq d_{p, a}-d_{\text {cov }}(p)$ is called the critical distance for the FAR node $a$ with respect to the primary transmitter $p$. Otherwise, $s_{a}^{*}=0 .^{2}$

\section{ROLE OF LOCALIZATION AND CRB}

Our proposed scheme for discovering spectrum holes is based on localizing the primary transmitters and using the location estimates to approximate the MIFTP. Localization in this context differs from more conventional scenarios (cf. [10]) in two respects: (1) The FAR nodes collaboratively localize the primary transmitter. (2) No cooperation is assumed between the FAR node and the primary transmitter. For the purpose of spectrum hole estimation, localization techniques based on the signal strength (SS) and angle-of-arrival (AOA) information are more appropriate than time-of-arrival (TOA) or timedifference-of-arrival (TDOA) methods. This is because in the noncooperative scenario, knowledge of the transmit waveform, which is required to extract the TOA information, is typically not available or difficult to obtain. For TDOA estimation, the conventional generalized cross-correlation method can be very demanding. This is because for a single TDOA estimate, the received (digitized) signals at two nodes need to be transmitted to a common site for processing, [11] p. 54.

\section{A. SS-based localization}

We limit our discussion to SS-based localization of a single primary transmitter in the geographic coverage area. Let $\boldsymbol{L}=\left[x_{p}, y_{p}\right]^{T}$ denote the location of the primary transmitter. Now suppose that a set of uncorrelated observed SS measurements, $\left\{S_{1}, \cdots, S_{N}\right\}$, is available, together with a corresponding set of position coordinates

\footnotetext{
${ }^{2}$ Due to space limitations, all proofs of original results in this paper are omitted, but can be found in [9].
} 
$\left\{\boldsymbol{L}_{1}, \cdots, \boldsymbol{L}_{N}\right\}$, where $\boldsymbol{L}_{i}=\left[x_{i}, y_{i}\right]^{T}, i=1, \cdots, N$. The set of observables,

$$
\mathcal{O} \triangleq\left\{\left(S_{i}, \boldsymbol{L}_{i}\right): i=1, \cdots, N\right\},
$$

may be obtained in several ways. For example, consider a scenario in which $N$ FAR nodes, located at positions $\boldsymbol{L}_{1}, \cdots, \boldsymbol{L}_{N}$, collect the signal strength observables $S_{1}, \cdots, S_{N}$ at a given time. The FAR nodes exchange their observables among each other, such that at least one of the FAR nodes receives the entire set $\mathcal{O}$. Such a FAR node can then compute an estimate $\hat{\boldsymbol{L}}=\left[\hat{X}_{p}, \hat{Y}_{p}\right]^{T}$ of the location of the primary transmitter. Alternatively, the observable set $\mathcal{O}$ may be obtained by measurements from a single FAR node at $N$ different points in time along a trajectory as the node moves in the coverage area. In general, a given observable $\left(S_{i}, \boldsymbol{L}_{i}\right)$ may be obtained either from a measurement taken by the FAR node itself in the past, or from a measurement by another FAR node that shares this information with the given FAR node.

Given a set of observations, $\mathcal{O}$, the observation equations can be written in vector form as follows:

$$
\boldsymbol{S}=\boldsymbol{z}+\boldsymbol{W},
$$

where

$$
\begin{aligned}
\boldsymbol{S} & =\left[S_{1}, \cdots, S_{N}\right]^{T}, \boldsymbol{z}=\left[z_{1}, \cdots, z_{N}\right]^{T}, \\
\boldsymbol{W} & =\left[W_{1}, \cdots, W_{N}\right]^{T},
\end{aligned}
$$

with $z_{i}=s_{p}-10 \epsilon \log _{10} d_{i}$, and $d_{i}=$ $\sqrt{\left(x_{i}-x_{p}\right)^{2}+\left(y_{i}-y_{p}\right)^{2}}$. An estimate $\hat{\boldsymbol{L}}$ of the location of the primary transmitter can be obtained from the SS observation equation (9).

\section{B. Cramér-Rao lower Bound}

The Cramér-Rao lower bound (CRB) provides a lower bound on the variance (or covariance matrix) of any unbiased estimate of an unknown parameter. For the SS localization model of (9), the CRB of any unbiased estimate $\hat{\boldsymbol{L}}$ of $\boldsymbol{L}$ is given by

$$
E_{\boldsymbol{L}}\left[(\hat{\boldsymbol{L}}-\boldsymbol{L})(\hat{\boldsymbol{L}}-\boldsymbol{L})^{T}\right] \geq \boldsymbol{J}_{\boldsymbol{L}}^{-1},
$$

where $E_{\boldsymbol{L}}[\cdot]$ denotes conditional expectation with respect to $\boldsymbol{L}$ and $\boldsymbol{J}_{\boldsymbol{L}}$ is the Fisher information matrix (FIM) given by

$$
\boldsymbol{J}_{\boldsymbol{L}}=E_{\boldsymbol{L}}\left[\frac{\partial}{\partial \boldsymbol{L}} \ln f_{\boldsymbol{S} \mid \boldsymbol{L}}(\boldsymbol{S})\left(\frac{\partial}{\partial \boldsymbol{L}} \ln f_{\boldsymbol{S} \mid \boldsymbol{L}}(\boldsymbol{S})\right)^{T}\right],
$$

where $f_{\boldsymbol{S} \mid \boldsymbol{L}}(\boldsymbol{S})$ is the likelihood function. In (12), the matrix inequality $\boldsymbol{A} \geq \boldsymbol{B}$ should be interpreted as the assertion that the matrix $\boldsymbol{A}-\boldsymbol{B}$ is non-negative definite. The CRB provides a lower bound on the mean-squared errors for the components of $\boldsymbol{L}$.

If the primary transmitter's signal power is known, the FIM can be expressed as follows [10]:

$$
\boldsymbol{J}_{\boldsymbol{L}}=\left(\frac{10 \epsilon}{\sigma_{W} \ln 10}\right)^{2} \boldsymbol{H} \boldsymbol{D}^{2} \boldsymbol{H}^{T},
$$

where

$$
\begin{aligned}
\boldsymbol{H} & \triangleq\left[\begin{array}{cccc}
\cos \phi_{1} & \cos \phi_{2} & \cdots & \cos \phi_{N} \\
\sin \phi_{1} & \sin \phi_{2} & \cdots & \sin \phi_{N}
\end{array}\right], \\
\boldsymbol{D} & \triangleq \operatorname{diag}\left[d_{1}^{-1}, \cdots, d_{N}^{-1}\right]
\end{aligned}
$$

and

$$
\phi_{i}=\tan ^{-1}\left(\frac{y_{p}-y_{i}}{x_{p}-x_{i}}\right), \quad i=1, \cdots, N,
$$

is the angle between $x$-axis and the line connecting $\left(x_{i}, y_{i}\right)$ and $\left(x_{p}, y_{p}\right)$ measured counterclockwise.

\section{Unknown Transmitter Power}

For opportunistic spectrum access, the transmit power is unknown. Therefore, the parameter vector to be estimated and its MLE are given by

$$
\boldsymbol{\Theta}=\left[x_{p}, y_{p}, s_{p}\right]^{T} \text { and } \hat{\boldsymbol{\Theta}}_{\mathrm{ML}}=\left[\hat{X}_{p}, \hat{Y}_{p}, \hat{S}_{p}\right]^{T},
$$

respectively. The next proposition gives a closed-form expression for the CRB when the transmit power is unknown.

Proposition 2: The CRB is given by

$$
\boldsymbol{J}_{\Theta}^{-1}=\left[\begin{array}{cc}
\boldsymbol{J}_{\boldsymbol{L}}^{-1}+b^{-1} \boldsymbol{c} \boldsymbol{c}^{T} & -b^{-1} \boldsymbol{c} \\
-b^{-1} \boldsymbol{c}^{T} & b^{-1}
\end{array}\right],
$$

where

$$
\begin{array}{r}
b \triangleq \frac{N}{\sigma_{W}^{2}}-\boldsymbol{a}^{T} \boldsymbol{J}_{\boldsymbol{L}}^{-1} \boldsymbol{a}, \quad \boldsymbol{c} \triangleq \boldsymbol{J}_{\boldsymbol{L}}^{-1} \boldsymbol{a}, \\
\boldsymbol{a} \triangleq-\frac{10 \epsilon}{\sigma_{W}^{2} \ln 10}\left[\sum_{i=1}^{N} \frac{\cos \phi_{i}}{d_{i}}, \sum_{i=1}^{N} \frac{\sin \phi_{i}}{d_{i}}\right]^{T},
\end{array}
$$

assuming that $\boldsymbol{J}_{\boldsymbol{L}}$ given in (13) is invertible.

\section{APPROXIMATION FOR MIFTP}

The true MIFTP, as given in Proposition 1, cannot be calculated directly, since the true location, $\boldsymbol{L}=$ $\left[x_{p}, y_{p}\right]^{T}$, of the primary transmitter $p$ is unknown. In this section, we derive an approximation for the MIFTP first for the case where the transmit power is known and then for the case in which it is unknown.

\section{A. Known transmit power}

Assume first that the transmit power $s_{p}$ of the primary transmitter is a known constant. Let $\hat{\boldsymbol{L}}_{\mathrm{ML}}=\left[\hat{X}_{p}, \hat{Y}_{p}\right]^{T}$ denote the MLE of $\boldsymbol{L}$. Given a set of $N \geq 3$ independent SS measurements from the primary transmitter, obtained by the FAR nodes, $\hat{\boldsymbol{L}}_{\mathrm{ML}}$ provides an unbiased estimate of $\boldsymbol{L}$ as the shadowing noise tends to zero. In this asymptotic regime, the mean squared error of $\hat{\boldsymbol{L}}_{\mathrm{ML}}$ achieves the CRB, which we denote by $J_{L}^{-1}$ [10].

Suppose that the FAR node $a$ is located at $\boldsymbol{L}_{a}=$ $\left[x_{a}, y_{a}\right]^{T}$. Given $\hat{\boldsymbol{L}}_{\mathrm{ML}}$, the MLE for the distance $d_{p, a}$, denoted by $\hat{D}_{p, a}$, can be obtained by applying the invariance principle (cf. [12], p. 217), which states that 


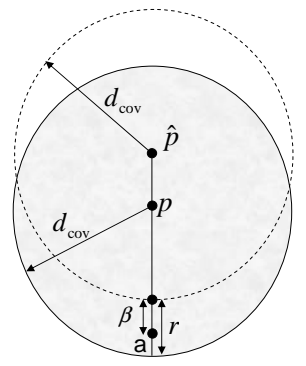

(a) $|r|>\beta$

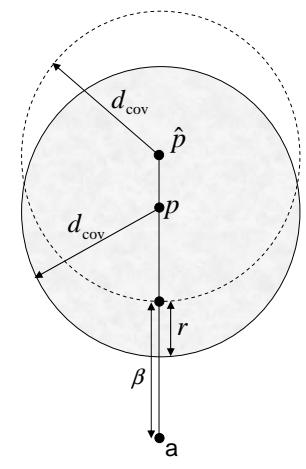

(b) $|r|<\beta$
Fig. 1. Illustration of relationship between $r$ and $\beta$. If $0<|r|<\beta$, $s_{a}^{*}>0$. Otherwise, $s_{a}^{*}=0$.

the MLE of a function $h(\cdot)$ of $\boldsymbol{L}$ is given by $h(\hat{\boldsymbol{L}})$, where $\hat{\boldsymbol{L}}$ denotes the MLE of $\boldsymbol{L}$. Hence, we obtain

$$
\hat{D}_{p, a}=\sqrt{\left(\hat{X}_{p}-x_{a}\right)^{2}+\left(\hat{Y}_{p}-y_{a}\right)^{2}} .
$$

Proposition 3: In the asymptotic regime $\sigma_{W}^{2} \rightarrow 0$, the MLE $\hat{D}_{p, a}$ achieves the associated CRB, given by

$$
J_{p, a}^{-1} \triangleq \boldsymbol{H}_{p, a}^{T} \boldsymbol{J}_{\boldsymbol{L}}^{-1} \boldsymbol{H}_{p, a}
$$

where

$\boldsymbol{H}_{p, a} \triangleq\left[\cos \phi_{p, a}, \sin \phi_{p, a}\right]^{T}, \phi_{p, a}=\tan ^{-1}\left(\frac{y_{p}-y_{a}}{x_{p}-x_{a}}\right)$.

Let $E_{p, a} \triangleq \hat{D}_{p, a}-d_{p, a}$ denote the estimation error of $\hat{D}_{p, a}$. Proposition 3 implies that in the asymptotic regime $\sigma_{W}^{2} \rightarrow 0, E_{p, a}$ is Gaussian with zero mean and variance $J_{p, a}^{-1}$, i.e.,

$$
E_{p, a} \sim \mathcal{N}\left(0, J_{p, a}^{-1}\right)
$$

Define $\beta \triangleq \hat{D}_{p, a}-d_{\text {cov }}(p)$. Suppose $E_{p, a}=r$. If $|r| \geq$ $\beta>0$, then in the worst case, the FAR node lies within $d_{\text {cov }}(p)$ of the true primary transmitter $p$ (see Fig. 1(a)). In this scenario, the FAR node must not transmit, i.e., $s_{a}^{*}=0$, to avoid potentially harmful interference to the victim nodes. If $0<|r|<\beta$, then the FAR can transmit with positive power, i.e., $s_{a}^{*}>0$ (see Fig. 1(b)). For this case, we obtain the following result.

Proposition 4: Under the assumption (22) and for $|r| \leq 0.993 \beta$, the interference probability conditioned on $E_{p, a}$ is upper bounded as follows:

$$
P_{\text {int }}\left(a, v \mid E_{p, a}=r\right) \leq Q\left(b_{1}+b_{2}|r|\right),
$$

where

$$
b_{1} \triangleq \frac{i_{\max }+10 \epsilon \log _{10} \beta-s_{a}}{\sigma_{W}}, b_{2} \triangleq-\frac{50 \epsilon}{\beta \sigma_{W} \ln 10} .
$$

Requiring that $s_{a}>0$, we obtain

$$
P_{\text {int }}(a, v)=\int_{-\beta}^{\beta} P_{\text {int }}\left(a, v \mid E_{p, a}=r\right) f_{E_{p, a}}(r) d r,
$$

where $f_{E_{p, a}}(r)$ denotes the probability density function (pdf) of $E_{p, a}$. We can then show that

$$
\begin{aligned}
P_{\text {int }}(a, v) & \leq \int_{-\infty}^{\infty} Q\left(b_{1}+b_{2} r\right) f_{E_{p, a}}(r) d r \\
& =Q\left(\frac{b_{1}}{\sqrt{1+b_{2}^{2} J_{p, a}^{-1}}}\right)
\end{aligned}
$$

where the last equality can be found in [13], p. 102.

To obtain an expression for the MIFTP, we require the FAR node transmitter power, $s_{a}$, to satisfy

$$
Q\left(\frac{b_{1}}{\sqrt{1+b_{2}^{2} J_{p, a}^{-1}}}\right) \leq \varepsilon_{\mathrm{int}} .
$$

Since the true CRB of $\hat{D}_{p, a}$, i.e., $J_{p, a}^{-1}$ is unknown, we replace it with the MLE of $J_{p, a}^{-1}$, which is denoted by $\hat{J}_{p, a}^{-1}$. This is justified by the invariance principle mentioned earlier and also illustrated in Section $\mathrm{V}$ in our numerical studies. Hence, we obtain the following approximation for the MIFTP:

$$
\begin{aligned}
\hat{s}_{a} & =i_{\max }+10 \epsilon \log _{10} \beta \\
& -\sigma_{W} \sqrt{1+\left(\frac{50 \epsilon}{\beta \sigma_{W} \ln 10}\right)^{2} \hat{J}_{p, a}^{-1}} \cdot Q^{-1}\left(\varepsilon_{\mathrm{int}}\right) .
\end{aligned}
$$

We point out that as the accuracy of the estimate $\hat{D}_{p, a}$ improves, the CRB estimate $\hat{J}_{p, a}^{-1}$ tends to zero and the right-hand side of (25) converges to the true MIFTP as given in (8). The approximate formula (25) for MIFTP requires at least three independent signal strength measurements, i.e., $N \geq 3$, which should be obtained from FAR nodes in the vicinity of the primary transmitter.

\section{B. Unknown transmit power}

When the primary transmitter power $s_{p}$ is not known, it can be estimated together with the location $\boldsymbol{L}$ as a parameter vector $\boldsymbol{\Theta}=\left[\boldsymbol{L}^{T}, s_{p}\right]^{T}$. We can show that the MLE, $\hat{\Theta}_{\mathrm{ML}}$, achieves the CRB asymptotically as $\sigma_{W}^{2} \rightarrow 0$ [9]. However, as shown by (17), the localization error increases due to the estimation of the additional parameter $s_{p}$. In particular, the CRB on location is given by

$$
J_{p, a}^{-1}=\boldsymbol{H}_{p, a}^{T}\left(\boldsymbol{J}_{\boldsymbol{L}}^{-1}+b^{-1} \boldsymbol{c} \boldsymbol{c}^{T}\right) \boldsymbol{H}_{p, a} .
$$

We define $\beta_{1} \triangleq \hat{D}_{p, a}-\hat{D}_{\operatorname{cov}}(p)$, where $\hat{D}_{\text {cov }}(p)=$ $g^{-1}\left(\hat{S}_{p}-r_{\min }+\sigma_{W} Q^{-1}\left(1-\varepsilon_{\mathrm{cov}}\right)\right)$. Let $E_{\mathrm{cov}} \triangleq \hat{D}_{\mathrm{cov}}(p)-$ $d_{\text {cov }}(p)$ denote the estimation error of $\hat{D}_{\text {cov }}(p)$. Similarly to $E_{p, a}$, we can model $E_{\text {cov }}$ as $E_{\text {cov }} \sim \mathcal{N}\left(0, J_{\text {cov }}^{-1}\right)$, where

$$
J_{\mathrm{cov}}^{-1}=\left(\frac{\partial d_{\operatorname{cov}}(p)}{\partial s_{p}}\right)^{2} b^{-1}=\left(\frac{d_{\operatorname{cov}}(p) \ln 10}{10 \epsilon}\right)^{2} b^{-1},
$$

and $b$ is given in (18). Defining $E \triangleq E_{p, a}-E_{\text {cov }}$, we see that $E \sim \mathcal{N}\left(0, J_{1}^{-1}\right)$, where $J_{1}^{-1} \triangleq J_{p, a}^{-1}+J_{\text {cov }}^{-1}-$ $2 \operatorname{Cov}\left(E_{p, a}, E_{\mathrm{cov}}\right)$. The covariance of $E_{p, a}$ and $E_{\mathrm{cov}}$ can 
be calculated by applying the same invariance principle used to obtain (21).

Let $E_{p, a}=r$ and $E_{\mathrm{cov}}=r_{0}$. The FAR node can transmit with positive power, i.e., $s_{a}^{*}>0$, if $0<\mid r-$ $r_{0} \mid<\beta_{1}$, otherwise $s_{a}^{*}=0$. Here, the critical distance from the FAR node is given by

$$
\begin{aligned}
d_{a}^{*} & =d_{p, a}-d_{\operatorname{cov}}(p)=\hat{D}_{p, a}-r-\left(\hat{D}_{\operatorname{cov}}(p)-r_{0}\right) \\
& =\beta_{1}-r_{1}
\end{aligned}
$$

where $r_{1} \triangleq r-r_{0}$. We note that here $r_{1}$ plays the same role as $r$ of Section IV-A.

Following the same steps as before, we can obtain the following MIFTP approximation for the case of unknown transmit power (cf. (25)):

$$
\begin{aligned}
\hat{s}_{a} & =i_{\max }+10 \epsilon \log _{10} \beta_{1} \\
& -\sigma_{W} \sqrt{1+\left(\frac{50 \epsilon}{\beta_{1} \sigma_{W} \ln 10}\right)^{2} \hat{J}_{1}^{-1}} \cdot Q^{-1}\left(\varepsilon_{\mathrm{int}}\right) .
\end{aligned}
$$

\section{Numerical Results}

In this section, we present plots of the true MIFTP and the approximate MIFTP estimated from signal strength measurements under a range of parameter settings. We choose our simulation parameters keeping in mind the application to unused television broadcast bands. We consider the case when $s_{p}$ is unknown because of its practical importance. The crucial parameters affecting the MIFTP estimation are $d_{p, a}, s_{p}, \varepsilon_{\text {int }}, \sigma_{W}, \epsilon, N$ and the CRB $J_{p, a}^{-1}$. We shall assume that the remaining parameters are known constants. Each of the MIFTP values is calculated as an average over 1000 simulation trials and is shown with the associated $95 \%$ confidence interval. We set the parameters as follows:

- Detection thresholds for the victim and FAR nodes are -83 and $-121 \mathrm{dBm}$, respectively.

- Path loss factor $\epsilon=4$. In practice, the path loss factor can be estimated separately [14], [15].

- $\varepsilon_{\mathrm{cov}}=0.05, i_{\mathrm{max}}=-100 \mathrm{dBm}, \varepsilon_{\mathrm{int}}=0.01$.

- Standard deviation of shadowing noise, $\sigma_{W}=8 \mathrm{~dB}$.

- Primary node location $\boldsymbol{L}=[50,50]^{T}[\mathrm{~km}]$.

\section{A. Distance $d_{p, a}$}

Fig. 2 plots the true and estimated MIFTP values vs. $d_{p, a}$ for $N=5,10,15,20$ for the case $\epsilon=4$. Here, we vary $d_{p, a}$ from 20 to $100 \mathrm{~km}$ and position the target FAR node at $\boldsymbol{L}_{a}=\left(x_{a}, y_{a}\right)$, where

$$
\boldsymbol{L}_{a}=\boldsymbol{L}+\frac{d_{p, a}}{\sqrt{2}}[1,1]^{T} .
$$

For a given transmit power of the primary transmitter, $s_{p}=80 \mathrm{dBm}$, we find $d_{\text {sense }}(a)$, the sensing distance of the FAR nodes. It denotes the radius beyond which the FAR nodes cannot detect the primary signal and is given by $d_{\text {sense }}(a)=g^{-1}\left(s_{p}-r_{a}+\sigma_{W} Q^{-1}\left(1-\varepsilon_{\text {cov }}\right)\right)$, where $r_{a}$ denotes the FAR node sensitivity. For each simulation trial, we randomly place $N$ FAR nodes, with uniform distribution, inside the circle with radius equal to $d_{\text {sense }}(a)$ and centered at $\boldsymbol{L}$. The set of SS measurements to compute the MLE of $\boldsymbol{L}$ or $\boldsymbol{\Theta}$ is collected by these FAR nodes, which can be used by other far away FAR nodes to estimate the MIFTP.

The confidence intervals shown in Fig. 2 arise due to randomness in the localizing FAR node positions, as well as the shadowing noise. We see that the accuracy of the approximate MIFTP formula improves with increasing $d_{p, a}$ and increasing $N$. In our simulations we found that the MIFTP values depend strongly on the path loss factor $\epsilon$. For larger values of $\epsilon$, the accuracy of the MIFTP approximation improves significantly and the effect of $N$ decreases. This is because, although the received signal becomes weaker as $\epsilon$ increases, the sensitivity of the MIFTP approximation to the location estimation error reduces.

In our simulation studies we have observed that, the MLE of the CRB can be estimated quite accurately for $N \geq 10$ and almost in all cases, it is greater than its true value. This justifies the use of the MLE of CRB in the MIFTP calculation. We can also calculate the probability of interference, $\hat{P}_{\text {int }}$, which results when the FAR node transmits with power level equal to the MIFTP estimate. Let $\hat{s}_{a}^{i}$ denote the MIFTP estimate for the $i$ th simulation trial, $i=1, \cdots, M$. Then the probability of interference under the MIFTP approximation is given by

$$
\hat{P}_{\text {int }}=\frac{1}{M} \sum_{i=1}^{M} P_{\text {int }}\left(a \mid \hat{s}_{a}^{i}\right),
$$

where $P_{\text {int }}\left(a \mid \hat{s}_{a}\right)$ denotes the interference probability given the FAR node transmits with power $\hat{s}_{a}$ (cf. (6)). Fig. 3 shows that $\hat{P}_{\text {int }}$ increases with increasing $d_{p, a}$, but is always less than $\varepsilon_{\text {int }}$. Therefore, the approximate MIFTP value can safely be used by the FAR node as an upper bound on the maximum allowable transmit power.

\section{B. Interference probability threshold, $\varepsilon_{\text {int }}$}

Fig. 4 shows a plot of MIFTP vs. the interference probability threshold, $\varepsilon_{\text {int }}$, which is varied from 0.001 to 0.1 . In this scenario, we set $\epsilon=4$, and $d_{p, a}=50$ $\mathrm{km}$. The location of the FAR node is set according to (28) and the values of the other parameters are set as in the previous scenario. We have observed that the MIFTP increases relatively slowly with increasing $\varepsilon_{\text {int }}$. In particular, for $N \geq 10$ there exists a tradeoff in the MIFTP value of about $15 \mathrm{~dB}$ when $\varepsilon_{\text {int }}=0.001$ and when $\varepsilon_{\text {int }}=0.1$. Also, the gap between the true and approximate MIFTP values decreases slowly with increasing $\varepsilon_{\text {int }}$. We observed that $\hat{P}_{\text {int }}$ increases almost linearly with increasing $\varepsilon_{\text {int }}$, but is always less than the specified threshold. These results suggest that the MIFTP approximation provides a tight approximate upper bound to the true MIFTP.

\section{CONCLUSiON}

The main result of this paper is an accurate method for estimating the maximum interference-free transmit 


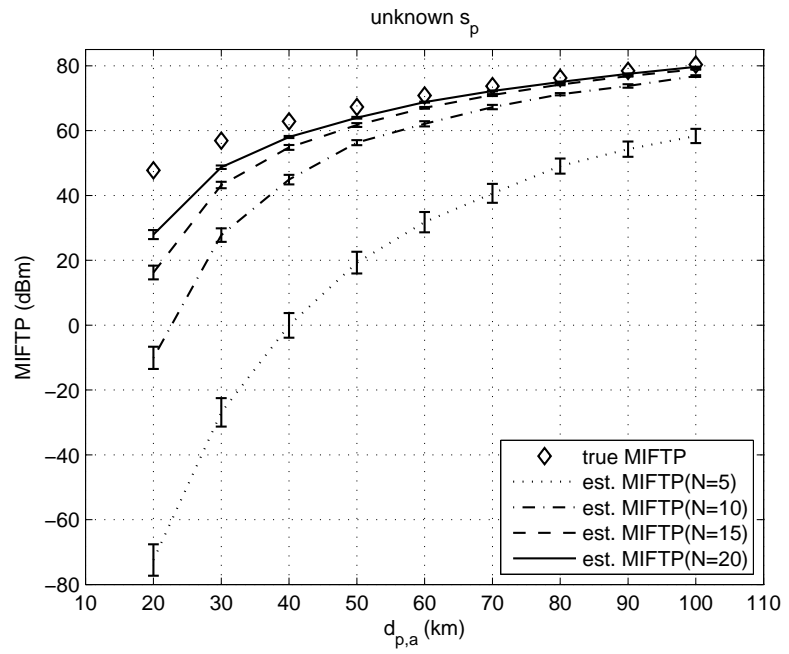

Fig. 2. MIFTP versus $d_{p, a}$.

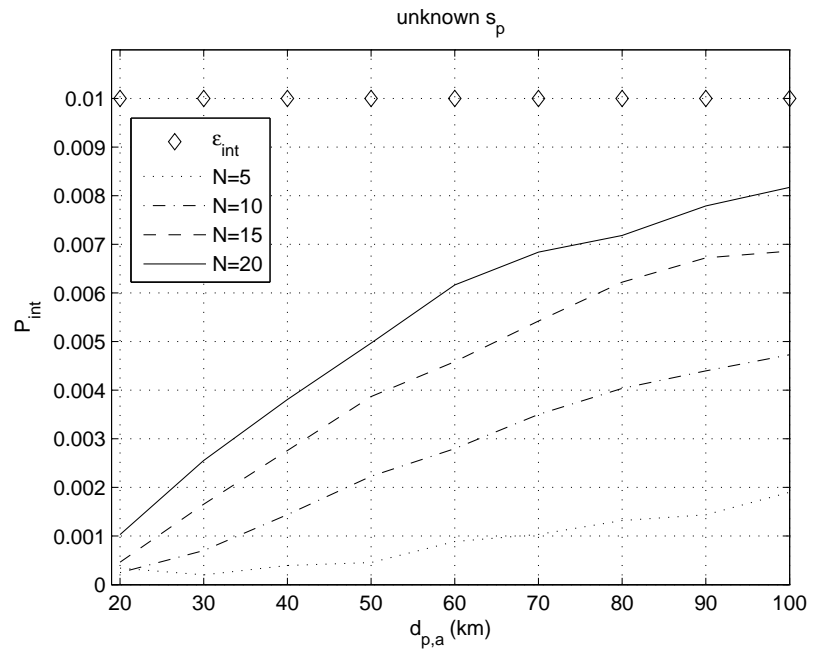

Fig. 3. $\hat{P}_{\text {int }}$ versus $d_{p, a}$.

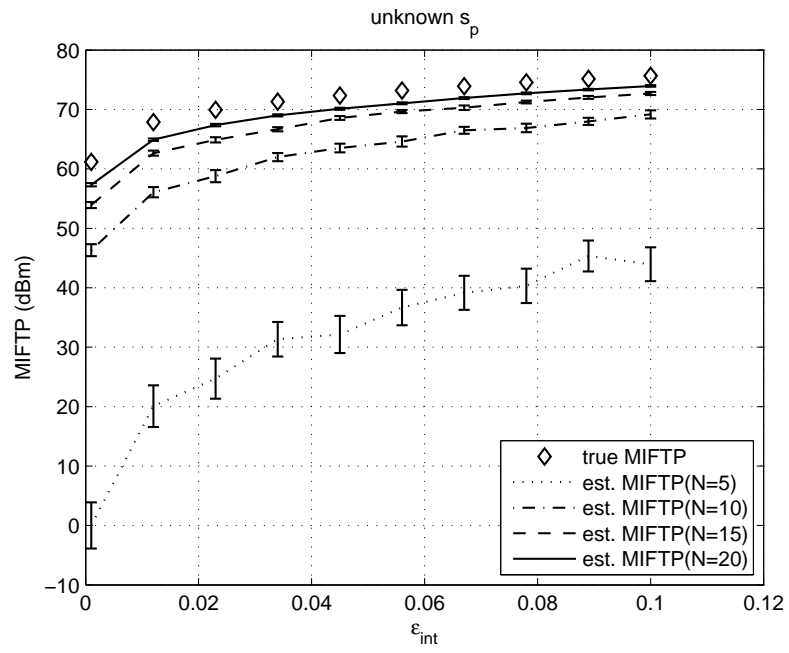

power (MIFTP), which a FAR can use in a given frequency channel without causing harmful interference to victim nodes. In effect, the MIFTP provides a concrete characterization of the size of a spectrum hole in the spatial domain for a given frequency channel. The MIFTP formula derived in this paper relies on signal strength measurements obtained by one or more FAR nodes in the vicinity of a given primary transmitter.

Our numerical results validate the accuracy of the proposed MIFTP estimation formula for several different scenarios and over a range of parameter settings. The proposed MIFTP estimation method can significantly enhance the performance of opportunistic spectrum access methods (cf. [8]), by exploiting collaboration among the FAR nodes. In ongoing work, we are exploring how other types of information gathered by the FAR nodes can be used to further improve the efficiency of spectrum hole estimation.

\section{REFERENCES}

[1] M. McHenry, "Frequency agile spectrum access technologies," in Proc. FCC Workshop on Cognitive Radio, May 2003.

[2] S. Haykin, "Cognitive Radio: Brain-Empowered Wireless Communications," IEEE J. Selected Areas in Comm., vol. 23, pp. 201220, Feb. 2005.

[3] S. Tang and B. L. Mark, "Performance analysis of a wireless network with opportunistic spectrum sharing," in Proc. IEEE Globecom, (Washington DC), Nov. 2007.

[4] Q. Zhao and A. Swami, "A decision-theoretic framework for opportunistic spectrum access," IEEE Communications Magazine: Special Issue on Cognitive Wireless Networks, vol. 14, pp. 14-20, Aug. 2007.

[5] R. Menon, R. M. Buehrer, and J. H. Reed, "Outage probability based comparison of underlay and overlay spectrum sharing techniques," in Proc. IEEE DySPAN, pp. 101-109, Nov. 2005.

[6] N. Hoven and A. Sahai, "Power scaling for cognitive radio," in Proc. Int. Conf. on Wireless Networks, Communications and Mobile Computing, pp. 250-255, June 2005.

[7] M. Gudmundson, "Correlation model for shadow fading in mobile radio systems," Electronics Letters, vol. 47, pp. 2145-2146, Nov. 1991.

[8] A. E. Leu, M. McHenry, and B. L. Mark, "Modeling and analysis of interference in listen-before-talk spectrum access schemes," Int. J. Network Mgmt, vol. 16, pp. 131-147, 2006.

[9] B. L. Mark and A. O. Nasif, "Estimation of Interference-Free Power for Opportunistic Spectrum Access," tech. rep., George Mason University, Sept. 2007.

[10] Y. Qi, H. Kobayashi, and H. Suda, "Analysis of Wireless Geolocation in a Non-Line-of-Sight Environment," IEEE Trans. on Wireless Comm., vol. 5, pp. 672-681, March 2006.

[11] J. J. Caffery, Wireless Location in CDMA Cellular Radio Systems. Massachusetts: Kluwer Academic Publishers, 1999.

[12] L. L. Scharf, Statistical Signal Processing: Detection, Estimation, and Time Series Analysis. New York: Addison-Wesley, 1991.

[13] S. Verdú, Multiuser Detection. New York: Cambridge University Press, 1998.

[14] V. Erceg et al., "An empirically based path loss model for wireless channels in suburban environment," IEEE J. Selected Areas in Comm., vol. 17, pp. 1205-1211, July 1999.

[15] C. Pérez-Vega and J. M. Zamanillo, "Path-loss model for broadcasting applications and outdoor communication systems in the VHF and UHF bands," IEEE Trans. on Broadcasting, vol. 48, pp. 91-96, June 2002.

Fig. 4. MIFTP versus $\varepsilon_{\text {int }}$. 